\title{
A LONG-TERM RADIOGRAPHIC EVALUATION OF GRAFT HEIGHT CHANGES AFTER MAXILLARY SINUS FLOOR AUGMENTATION AND SIMULTANEOUS IMPLANT PLACEMENT
}

\author{
Alshaimaa Ahmed Shabaan*, Mohammed Abdelsalam El-Baz** \\ and Inass Abdel Rahman AbuElmagd ${ }^{* * * *}$
}

\begin{abstract}
Objective: The aim of this study was to evaluate the long-term changes in the vertical bone height after sinus augmentation and simultaneous implant placement.

Patients and Method: Twenty-nine patients had received a sinus floor elevation with simultaneously inserted implants. The sinus floor elevation was performed through a lateral window osteotomy. The implant sites were prepared using the conventional drilling technique. The vertical bone height was measured from immediately, 6 months, 1 year and 5 years postoperative radiographs.
\end{abstract}

Results: A significant reduction of vertical bone height occurred during the first 12 months after which the resorption rate slow down and bone height eventually became stable $(\mathrm{P}<0.001)$.

Conclusion: Sinus augmented sites showed a radiographic reduction of the vertical height over the first year and minimal changes had been observed in the 5-year period following maxillary sinus floor elevation.

KEY WORDS: Sinus floor elevation, bone grafting, dental implant, long term observation, vertical bone height.

\section{INTRODUCTION}

Periodontal disease and dental caries are the main causes of tooth loss and the incidence of edentulous patients varies worldwide between $7 \%$ and $69 \%{ }^{(1)}$. Complete or partial tooth loss is often associated with nutritional deficiencies, oral pain, and poor psychosocial functioning.

During the last 40 years, osseointegrated dental implants have become one of the most used biomaterial to replace missing or lost teeth and the treatment has been characterized by a

\footnotetext{
* Assistant Professor of Oral \& Maxillofacial Surgery, Faculty of Dentistry, Fayoum University

** Lecturer of Oral \& Maxillofacial Surgery, Faculty of Dentistry, MSA University.

*** Lecturer of Oral Radiology, Faculty of Dentistry, Fayoum University
} 
highly successful outcome of complete, partial or single edentulism ${ }^{(2-5)}$. Oral rehabilitation with implant-supported prosthesis have shown improved masticatory function and oral specific healthrelated quality of life compared to removable dentures $^{(6,7)}$. However, placement of implants in the posterior part of the maxilla is frequently compromised or impossible due to atrophy of the alveolar process, poor bone quality and maxillary sinus pneumatization. Therefore, vertical alveolar ridge augmentation is often necessary before or in conjunction with installation of implants.

Various surgical approaches comprising elevation of the Schneiderian membrane have been proposed to achieve the necessary vertical height of the alveolar process for the installation of implants with a sufficient length including maxillary sinus floor augmentation with the lateral window technique, osteotome-mediated sinus floor elevation ${ }^{(8-10)}$. However, the treatment of choice for the most appropriate surgical intervention for oral rehabilitation of the atrophic posterior maxillary ridge with implants is influenced by the vertical height of the residual alveolar bone, local intrasinus anatomy and the number of teeth to be replaced ${ }^{(10)}$.

Different types of biomaterials have been used for maxillary sinus floor augmentation including autograft, allograft, xenograft, alloplast, and growth factors, and the selection of the ideal graft material has been a subject of controversy over the years.

However, augmented sites were subjected to a conversion processes over time, which can lead to a considerable decrease in bone over a time after augmentation $^{(11-14)}$

Long-term graft stability is essential for incorporating dental implants with a good prognosis. Bio-oss showed slow resorption rate that allow better volumetric stability and resist the repnumatization of the maxillary sinus. It tent up the elevated sinus membrane, thus maintaining the physical dimension of the graft In comparative studies, the volumetric stability of the graft with Bio-Oss, or different ratios of Bio-Oss and autogenous bone, has been assessed after 12 weeks in minipigs using unbiased threedimensional quantitation of computed tomography Images. The volumetric stability of the graft was significantly influenced by the ratio of Bio-Oss and autogenous bone. ${ }^{(11-14,26)}$

Only a few studies have directly investigated the long-term stability of bone augmentations and vertical bone changes with an adequate follow-up. To date, there is no evidence regarding the longterm stability of bone grafts; long-term studies are needed to investigate changes in bone height and implant survival rate when augmentation is performed prior to implant insertion. ${ }^{(11-14)}$

The aim of this study was to evaluate the longterm changes in the vertical bone height after sinus augmentation and simultaneous implant placement and implant survival in a 5-year longitudinal study.

\section{PATIENTS AND METHODS}

\section{Participants}

Forty implants were placed in a total of 29 patients participated in this study, ranging in age from 30 to 56 years (mean $41.7 \pm 7.35$ ). They were seeking fixed prosthetic restoration for their posterior maxilla. Cases with a limited bone height below the floor of the maxillary sinus were particularly selected for this study. The sinus augmentation procedures

The participants were recruited from Outpatient clinics, Faculty of Dentistry, Cairo University and followed from 2012 through 2017. Patient selection was based on certain inclusion and exclusion criteria.

The inclusion criteria include that patients must have a maxillary partial (unilateral or bilateral) edentulism involving the premolar/molar areas and the presence of 5-7 mm crestal bone between the sinus floor and alveolar ridge. While exclusion criteria include smoking, patients with systemic diseases, maxillary sinus pathology, and those with recent extractions (less than 1 year) in the involved area. 
Completed medical and dental history was taken from all patients and alveolar bone was evaluated using CBCT. Patients were fully informed about the treatment procedures, follow-up examinations and complications of surgical procedures. Informed consent was obtained from each patient prior to participation in the study. Study was conducted in accordance with Helsinki Declaration of 1975 for medical studies as revised in 2000 .

All Patients were subjected to a standardized surgical protocol by the same surgeon and another operator carried out the postoperative measurements

\section{Surgical Procedure}

Before surgery, the mouths were rinsed with a chlorhexidine digluconate solution $0.2 \%$ for 2 minutes. With the patient under local anesthesia, a crestal incision was performed slightly palatally, supplemented with 2 buccal releasing incisions, mesially and distally. Full thickness flaps were elevated to expose the alveolar crest and lateral wall of the maxillary sinus.

A large bony window was created in the lateral wall of the maxillary sinus. The osteotomy at the inferior aspect of the window was made at or as close to the level of superior aspect of residual alveolar bone height. Cutting was applied in a light staccato fashion to strip away the outer bony cortex without damaging the Schneider membrane Fig (1).

The sinus membrane was elevated with curettes of different shapes until it became completely detached from the lateral and inferior wall of the sinus Fig (1).

Once the sinus lifting procedure is completed implants (Swiss Plus Implant System, Zimmer Dental Inc, Aston Avenue, USA) were placed according to the manufacturer's surgical protocol.

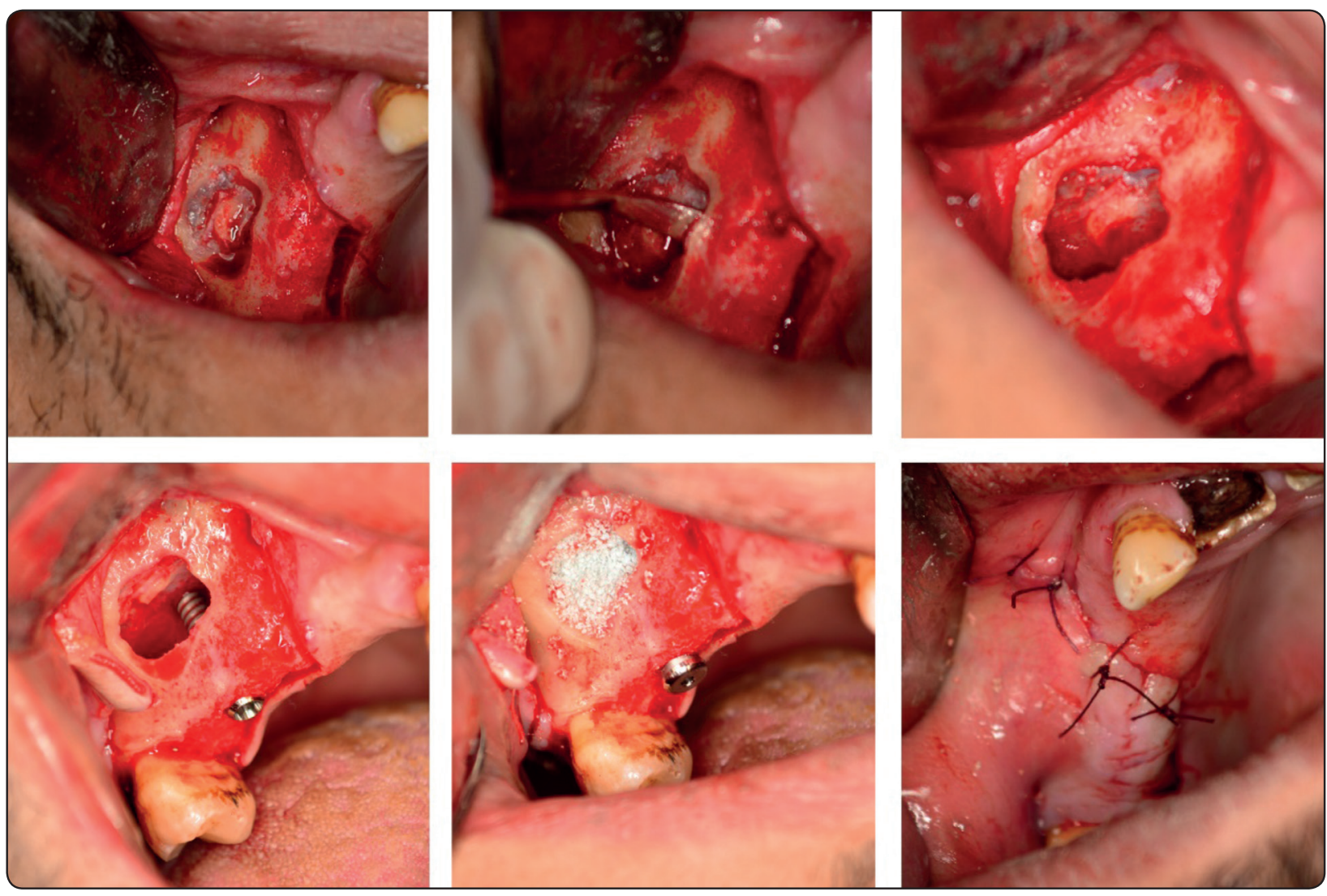

Fig. (1) Sinus membrane elevation procedures 
Thereafter the grafting materials (Bio-oss, Geistlich pharma AG, Wholhusen, Switherland) were mixed with saline and carefully packed in the sinus cavity. Flaps were sutured.

The patients received detailed verbal and written postoperative instructions. Antibiotic therapy consisting of $1 \mathrm{~g}$ amoxicillin (Augmentin $1 \mathrm{gm}$, Smithline Beecham Pharmaceutical Co., Bentford, England) every $12 \mathrm{~h}$ for 4 days and mouth rinsing with $0.2 \%$ chlorhexidine (Hexitol mouth wash, the Arab Drug Co., Cairo, Egypt) every 8 h for 10 days were prescribed. The suture was removed one week postoperatively.

\section{Radiographic evaluation:}

The radiographic evaluation has been carried out using a standard CBCT (Scanora3DX, Sordex, Tuusula, Finland). The area of interest was identified, and axial correction of the view was performed in conformity with angulation of the alveolar ridge.

All the measurement of vertical bone height was performed using software of Ondemand 3D (ondemand3D software, Cyber Med, Korea.). For this purpose, Patients during the postoperative follow-up received CBCT immediately after the implant placement, 6 months, 1 year and 5 years postoperatively.
Linear measurements were taken by the same calibrated examiner. The vertical bone height was measured for each augmented implant site from the implant neck area in contact with crestal bone to the most apical extent of bone augmentation at the mesial, distal and mid-center areas and averaged to determine the total vertical bone height (Fig. 2)

\section{Statistical Analysis:}

Data were fed to the computer and analyzed using IBM SPSS software package version 20.0. Data were fed to the computer and analyzed using IBM SPSS software package version 20.0. (Armonk, NY: IBM Corp). The Kolmogorov- Smirnov, Shapiro and D'agstino tests were used to verify the normality of distribution of variables, Comparisons between groups for categorical variables were assessed using Chi-square test (Fisher or Monte Carlo). Student t-test was used to compare two groups for normally distributed quantitative variables. Mann Whitney test was used to compare between two groups for abnormally distributed quantitative variables. Significance of the obtained results was judged at the $5 \%$ level.

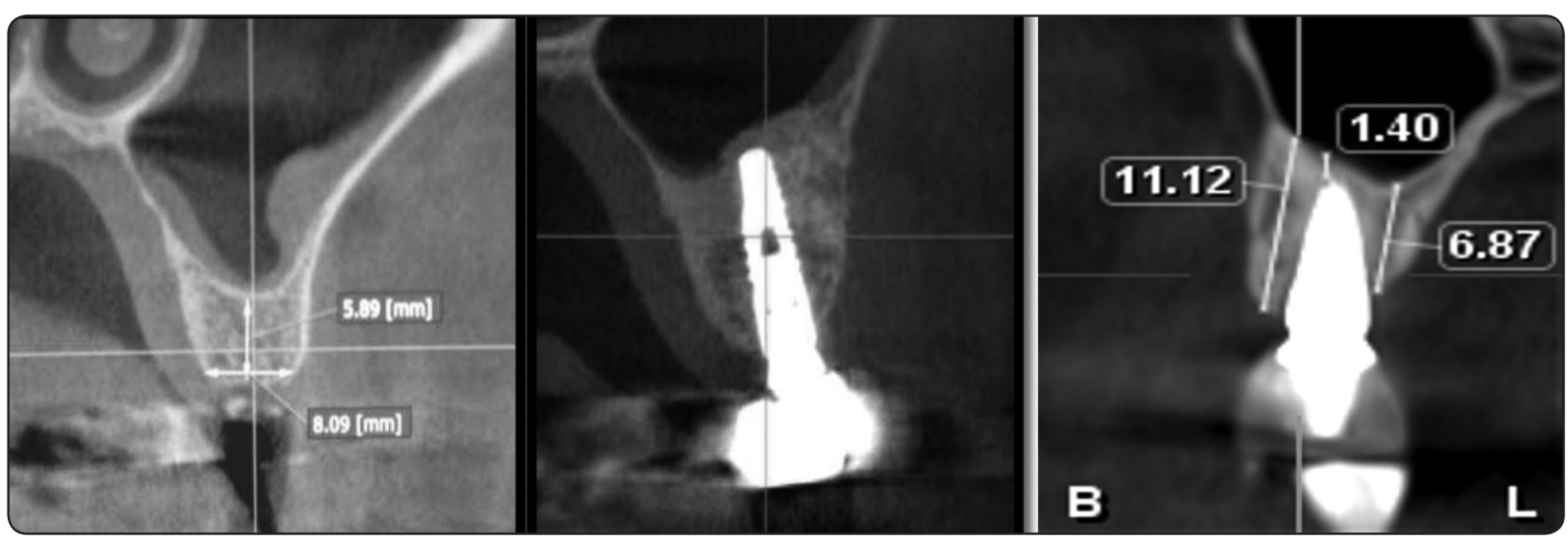

Fig. (2) The radiographic measurements of the alveolar ridge height 


\section{RESULTS}

A total of 40 implants were placed in a total of 29 patients participated in this study, ranging in age from 30 to 56 years (mean $41.7 \pm 7.35$ ). The height of bone in the selected cases range from 4.8 to 6.1 (mean $5.41 \pm 0.42$ ).

Forty implants were placed in the maxillary posterior teeth, twenty-nine implants $(72.3 \%)$ at the molar region and elven $(27.7 \%)$ in premolar region The patients received implants with mean length $10.83 \pm 1.023$ (Range: $10-12 \mathrm{~mm}$ ). All the placed implants are $3.7 \mathrm{~mm}$ diameter.

The surgical procedure was performed without any complication in all patients except for 2 cases

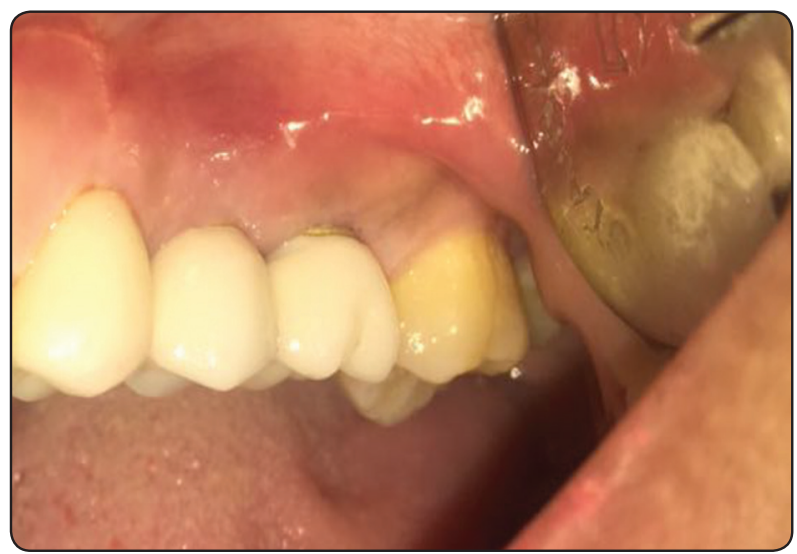

Fig. (3) Final restoration $2^{\text {nd }}$ premolar $\& 1^{\text {st }}$ molar that showed perforation in the sinus membrane and were excluded from the study. All implants healed without any complications until the final follow up visits. No signs of peri-implantitis were recorded throughout the 5 years follow-up period. Moreover, no patients reported any adverse actions such as pain or altered sensation in the treated areas during follow up period. The definitive restorations were porcelain fused to metal cement retained that were delivered 4- 6 months after implant placement. (fig. 3 )

The grafted height of the alveolar ridge was measured from the CBCT sagittal and coronal view on intervals of immediate, $6^{\text {th }}$ month, 1 year and 5 years postoperatively.

The mean height of grafted alveolar ridge in the immediate postoperative was $8.166 \pm 1.29$ and at the $6^{\text {th }}$ month postoperative interval was $7.25 \pm 0.91$. In the subsequent follow up interval, the height of the graft remained unchanged and measured $7.033 \pm 0.86$ in the $1^{\text {st }}$ year and $5^{\text {th }}$ year postoperative. (table $1 \&$ Fig. 4)

The mean height of grafted alveolar ridge had no statistical significant difference in the following postoperative intervals $\left(6^{\text {th }}\right.$ month, $1^{\text {st }}$ year and $5^{\text {th }}$ year postoperative) when compared with the immediate postoperative measurements. (table 2)

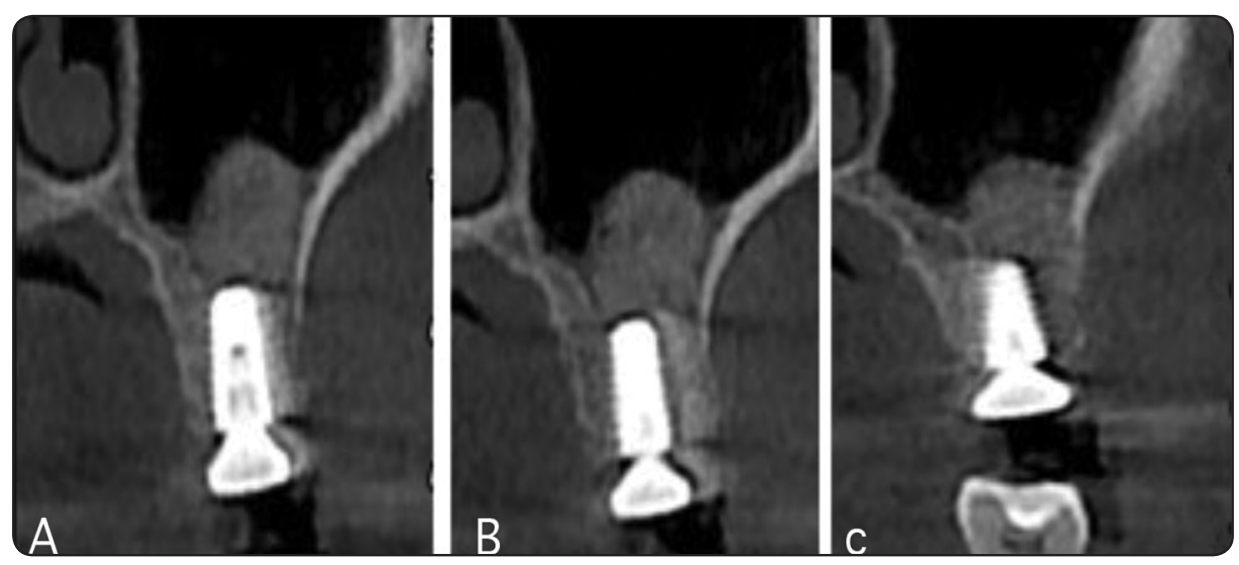

Fig. (4) Radiographic follow up a- $6^{\text {th }}$ month postoperative b- 1- year postoperative c- 5- years postoperative follow-up 
TABLE (1) The mean height of grafted alveolar ridge in mm immediately after grafting and postoperatively

\begin{tabular}{|l|c|c|c|}
\hline $\mathrm{N}=40$ & Minimum & Maximum & Mean \\
\hline Height of graft immediate & 6.31 & 10.13 & $8.166 \pm 1.29$ \\
\hline Height of graft at 6 months postoperative & 6.1 & 8.9 & $7.25 \pm 0.91$ \\
\hline Height of graft 1 year postoperative & 6 & 8.4 & $7.033 \pm 0.86$ \\
\hline Height of graft 5 years postoperative & 6 & 8.4 & $7.033 \pm 0.86$ \\
\hline
\end{tabular}

TABLE (2) Comparison of the mean height of grafted alveolar ridge between the follow up intervals

\begin{tabular}{|l|c|c|}
\hline (I) Group & (J) Group & Sig* \\
\hline Imm & $6 \mathrm{M}$ & 0.306 \\
& $1 \mathrm{Y}$ & 0.119 \\
& $5 \mathrm{Y}$ & 0.110 \\
\hline $6 \mathrm{M}$ & $\mathrm{Imm}$ & 0.306 \\
& $1 \mathrm{Y}$ & 0.992 \\
& $5 \mathrm{Y}$ & 0.992 \\
\hline $1 \mathrm{Y}$ & $\mathrm{Imm}$ & 0.119 \\
& $6 \mathrm{M}$ & 0.992 \\
& $1 \mathrm{Y}$ & 1.00 \\
\hline $5 \mathrm{Y}$ & $\mathrm{Imm}$ & 0.119 \\
& $6 \mathrm{M}$ & 0.992 \\
& $1 \mathrm{Y}$ & 1.00 \\
\hline
\end{tabular}

*The mean difference is significant at the .05 level.

\section{DISCUSSION}

Rehabilitation of the partially edentulous posterior maxilla with dental implants has proven to be a challenge for the dental implant team due to a deficiency of available bone volume, especially vertical bone height. Atrophy observed in the posterior maxilla is due to progressive bone resorption that accompanies tooth loss, pneumatization of the maxillary sinus, and age over time. This pneumatization process is accompanied by crestal bone loss that also prevents implant placement. ${ }^{(4-6)}$
The successful outcome of any implant procedure needs a series of patient-related parameters such as bone volume and quality besides proceduredependent parameters such as type of implant and surgical procedures. The importance of the implant and graft stability in long term clinical success is also well recognized. ${ }^{(5,15-17)}$

However, many studies have reported radiopacity upon radiographic examination of the bone regenerate in the augmented sites was flatter or concave at $6^{\text {th }}$ month postoperatively; this could be attributed to the laminar flow effect of air going in and out the sinus, thus massaging the upper surface of the regenerated. This phenomenon may affect the long-term survival rate of the dental implants placed in the augmented sinus. ${ }^{(18-20)}$

The aim of present study was to evaluate the long-term changes in the vertical bone height after sinus augmentation and simultaneous implant placement.

The present study examined the changes in bone height after augmentation using sinus elevation lateral window approach with a follow-up period of 5 years. All included patients had atrophy of the edentulous or partially edentulous jaw and received an augmentation with the sole use of Bio-oss bone graft.

Bone reduction in grafted areas for all patients, measured as loss in total bone height, initially decreased significantly and then the rate of resorption 
slowed down over time with no statistical significant in the $6^{\text {th }}$ month, $1^{\text {st }}$ year and $5^{\text {th }}$ year postoperative when compared with the immediate postoperative measurement.

This agreed with the results of Zijderveld et al. ${ }^{(12)}$ who investigated the long-term changes in graft height after maxillary sinus floor elevation with autologous bone from the chin (group 1) and 100\% beta-tricalcium phosphate (group 2) with a followup of 4.5 years. The radiographic evaluation showed no differences between the grafting groups over time. They reported an initial bone height reduction in the first 1.5 years. After 1.5 years, changes were minimal and not statistically significant.

Another study Wiltfang et al. ${ }^{(11)}$ also investigated the changes in bone height after sinus floor elevation and onlay grafting with iliac bone with a five-year follow-up. After 12 months, initial bone loss of $20 \%$ was demonstrated using the onlay grafting method and $17 \%$ using sinus elevation; the five-year followup reported no further decrease in bone height following 1 year after augmentation.

Furthermore, Starch et al. ${ }^{(21)}$ in their systematic review to the maxillary sinus augmentation concluded that the reduction in the vertical bone height after augmentation was significant during the first year, after which the resorption decreased.

This could be explained by the healing process after bone graft transplantation is takes place in different successive steps. First, resorptive processes dominate in the context of inflammation. Afterword's, the graft is vascularized, and the proliferating cells can penetrate the bone graft. The transplanted bone is resorbed and replaced successively with new bone. (22-25)

Applied to this study results, this process seems to lead to decreased bone volume, which might explain the initial bone resorption in the first 12 months. After the initial bone resorption, the regenerated bone shows the same biological behavior as nonregenerated natural bone and will remain stable even after 10 years. ${ }^{(22-25)}$

\section{CONCLUSION}

In conclusion, the results of the study showed that sinus augmented sites showed a radiographic reduction of the vertical height over the first year and minimal changes had been observed in the 5-year period following maxillary sinus floor elevation.

\section{Conflicts of Interests}

The authors declare that there are no competing interests regarding the publication of this paper.

\section{REFERENCES}

1. Petersen PE, Bourgeois D, Bratthall D, Ogawa H. Oral health information systems--towards measuring progress in oral health promotion and disease prevention. Bull World Health Organ. 2005; 83:686-93.

2. Berglundh T, Persson L, Klinge, BA. A systematic review of the incidence of biological and technical complications in implant dentistry reported in prospective longitudinal studies of at least 5 years. J Clin Periodontol. 2002; 29:197-212.

3. Pjetursson BE, Thoma D, Jung R, Zwahlen M, Zembic A. A systematic review of the survival and complication rates of implant-supported fixed dental prostheses (FDPs) after a mean observation period of at least 5 years. Clin Oral Implants Res. 2012; 23:22-38.

4. Jung RE, Zembic A, Pjetursson BE, Zwahlen M, Thoma DS. Systematic review of the survival rate and the incidence of biological, technical, and aesthetic complications of single crowns on implants reported in longitudinal studies with a mean follow-up of 5 years. Clin Oral Implants Res. 2012; 23:2-21.

5. Buser D, Sennerby L, De Bruyn H. Modern implant dentistry based on osseointegration: 50 years of progress, current trends and open questions. Periodontol 2000. 2017; 73:7-21.

6. Emami E, Heydecke G, Rompré PH, de Grandmont P, Feine JS. Impact of implant support for mandibular dentures on satisfaction, oral and general health-related quality of life: a meta-analysis of randomized-controlled trials. Clin Oral Implants Res. 2009; 20:533-44.

7. Boven GC, Raghoebar GM, Vissink A, Meijer HJ. Improving masticatory performance, bite force, nutritional state 
and patient's satisfaction with implant overdentures: a systematic review of the literature. J Oral Rehabil. 2015; 42:220-33.

8. Carreño Carreño J, Aguilar-Salvatierra A, Gómez-Moreno G, García Carreño EM, Menéndez López-Mateos ML, Perrotti V, Piattelli A, Calvo-Guirado JL, Menéndez-Núñez M. Update of Surgical Techniques for Maxillary Sinus Augmentation: A Systematic Literature Review. Implant Dent. 2016; 25:839-844.

9. Danesh-Sani SA, Loomer PM, Wallace SS. A comprehensive clinical review of maxillary sinus floor elevation: anatomy, techniques, biomaterials and complications. Br J Oral Maxillofac Surg. 2016; 54:724-30.

10. Lundgren S, Cricchio G, Hallman M, Jungner M, Rasmusson L, Sennerby L. Sinus floor elevation procedures to enable implant placement and integration: techniques, biological aspects and clinical outcomes. Periodontol 2000. 2017; 73:103-20.

11. Wiltfang, J., Schultze-Mosgau, S., Nkenke, E., Thorwarth, M., Neukam, F.W. \& Schlegel, K.A. Onlay augmentation versus sinuslift procedure in the treatment of the severely resorbed maxilla: a 5-year comparative longitudinal study. International Journal of Oral and Maxillofacial Surgery 2005;34: 885-889.

12. Zijderveld, S.A., Schulten, E.A., Aartman, I.H. \& ten Bruggenkate, C.M. Long-term changes in graft height after maxillary sinus floor elevation with different grafting materials: Radiographic evaluation with a minimum followup of 4.5 years. Clinical Oral Implants Research 2009;20: 691-700.

13. Simion, M., Fontana, F., Rasperini, G. \& Maiorana, C. Long-term eval uation of osseointegrated implants placed in sites augmented with sinus floor elevation associated with vertical ridge augmentation: a retrospective study of 38 consecutive implants with 1- to 7-year follow-up. International Journal of Periodontics and Restorative Dentistry 2004;24: 208-221.

14. Rocchietta, I., Fontana, F. \& Simion, M. Clinical outcomes of vertical bone augmentation to enable dental implant placement: a systematic review. Journal of Clinical Periodontology 2008;35:203-215.

15. Beer A, Gahleitner A, Holm A, Tschabitscher M, Homolka P. Correlation of insertion torques with bone mineral density from dental quantitative CT in the mandible. Clin Oral Implants Res. 2003; 14:616-20.

16. Ekfeldt A, Christiansson U, Eriksson T, Lindén U, Lundqvist S, Rundcrantz T, Johansson LA, Nilner K, Billström
C. A retrospective analysis of factors associated with multiple implant failures in maxillae. Clin Oral Implants Res. $2001 ; 12: 462-7$

17. Turkyilmaz I, Aksoy U, McGlumphy EA. Two alternative surgical techniques for enhancing primary implant stability in the posterior maxilla: a clinical study including bone density, insertion torque, and resonance frequency analysis data. Clin Implant Dent Relat Res. 2008; 10:231-7.

18. Jensen T, Schou S, Svendsen PA, Forman JL, Gundersen HJ, Terheyden H, Holmstrup P. Volumetric changes of the graft after maxillary sinus floor augmentation with Bio-Oss and autogenous bone in different ratios. A radiographic study in minipigs. Clin Oral Implants Res. 2012; 23:902-10.

19. Shanbhag S, Shanbhag V, Stavropoulos A. Volume changes of maxillary sinus augmentations over time: a systematic review. Int J Oral Maxillofac Implants. 2014; 29:881-92.

20. Jensen T, Schou S, Stavropoulos A, Terheyden H, Holmstrup P. Maxillary sinus floor augmentation with Bio-Oss or Bio-Oss mixed with autogenous bone as graft: a systematic review. Clin Oral Implants Res. 2012; 23:263-73.

21. Starch-Jensen T, Aludden H, Hallman M, Dahlin C, Christensen AE, Mordenfeld A. A systematic review and metaanalysis of long-term studies (five or more years) assessing maxillary sinus floor augmentation. Int J Oral Maxillofac Surg. 2018;47:103-116.

22. Binger, T. \& Hell, B. Resorption of microsurgically vascularized bone grafts after augmentation of the mandible. Journal of Cranio-maxillo-Facial Surgery 1999;27: 82-85.

23. Brugnami, F., Caiazzo, A. \& Leone, C. Local intraoral autologous bone harvesting for dental implant treatment: Alternative sources and criteria of choice. The Keio journal of medicine 2009;58: 24-28.

24. Buckwalter, J.A., Glimcher, M.J., Cooper, R.R. \& Recker, R. Bone biology. Ii: formation, form, modeling, remodeling, and regulation of cell function. Instructional Course Lectures 1996;45:387-399.

25. Burchardt, H. The biology of bone graft repair. Clinical Orthopaedics and Related Research 1983;174: 28-42.

26. Pasquali PJ, Teixeira ML, de Oliveira TA, de Macedo LG, Aloise AC, Pelegrine AA. Maxillary Sinus Augmentation Combining Bio-Oss with the Bone Marrow Aspirate Concentrate: A Histomorphometric Study in Humans. Int J Biomater. 2015; 2015:121286 